\title{
Fast Synergetic Control for Chaotic Oscillation in the Power System Based on Input-Output Feedback Linearization
}

\author{
Ling Liu, Jiangbin Wang ${ }^{D}$, and Chongxin Liu \\ State Key Laboratory of Electrical Insulation and Power Equipment, School of Electrical Engineering, Xi'an Jiaotong University, \\ Xi'an 710049, China \\ Correspondence should be addressed to Jiangbin Wang; 1550151867@qq.com
}

Received 9 June 2021; Accepted 11 July 2021; Published 20 July 2021

Academic Editor: Yuqiang Wu

Copyright $(92021$ Ling Liu et al. This is an open access article distributed under the Creative Commons Attribution License, which permits unrestricted use, distribution, and reproduction in any medium, provided the original work is properly cited.

This paper presents a fast synergetic control scheme for chaotic oscillation in a three-bus power system model. First, the coupling dynamic model of a controlled power system with the current source converter-based STATCOM device and energy storage device is established. Then, the input-output linearization process for the controlled power system is derived step by step, the control problem for the complex nonlinear power system model is completely transformed into the control of linear systems, and a fast synergetic control scheme is proposed for these linear systems. Since the designed control inputs contain complex system functions which are very difficult to obtain and reduce the engineering practicability of the designed controllers, the assumption that system functions are bounded is introduced into the controller design process, and the controllers are redesigned. The remarkable advantages of the proposed control method are that it improves the rapidity of traditional synergetic control and avoids complex system functions in control inputs. Finally, the effectiveness and the superiority of the control scheme are verified by simulation results.

\section{Introduction}

Power system is a typical high-dimensional, complex, and strong coupling dynamic system. Just as many other nonlinear systems exhibit bifurcation, chaos, and other complex nonlinear dynamic behaviors, so do power systems [1-5]. In recent years, scholars have studied how to effectively control chaos in the power system and obtained rich research results [6-8]. At present, the focus of research is to analyze and control chaotic oscillation in a three-bus power system [8-15]. Different from controlling chaos in some simple chaotic systems, the chaos control problem of the power system has obvious physical background. However, the control methods in [12-14] often fail to consider the physical background of the power system and control chaos by means of full-state regulation, which makes the designed control inputs not practical.

From the perspective of the power system, the problem of chaos control in the power system does not belong to a chaos control but a power system control problem, and controlling the power system needs to introduce control devices. Among numerous power system control devices, flexible alternating current transmission system (FACTS) devices have superior performance and can provide many superior options [16, 17]. However, the existing chaos control methods for the power system always ignore the use of the FACTS device to control chaotic oscillation in the power system [9-14]. Among the members of FACTS devices, unified power flow controller (UPFC) device has rich control functions and the most excellent performance, and it can adjust active power and reactive power at the same time. However, the structure of the device is complex, and the control input variables are numerous [18-20]; then, it is not conducive to design controllers. Since parallel devices in FACTS devices are easier to implement than series devices, static VAR compensator (SVC) device and static synchronous compensator (STATCOM) device have been widely used in power system control as two typical parallel devices [21-25]. Compared with the SVC device, the STATCOM device is superior in many technical performances $[26,27]$; 
then, the STATCOM device becomes the best choice among these control devices. At present, STATCOM devices have been widely used for reactive compensation and voltage regulation in the power system $[24,25]$. There are two types of STATCOM devices: voltage source converter-based device and current source converter-based device. Although the technology of the former is more mature and it is widely used as pointed out in $[28,29]$, compared with voltage source converter-based STATCOM, current source converter-based STATCOM has more application potential due to its advantages of less harmonic injection. STATCOM device is mainly used for reactive power control of the power system, and it is often necessary to control active power by introducing the energy storage device [30]. Therefore, it has practical value to design controllers for the chaotic power system by combining the current source converter-based STATCOM with the energy storage device.

To control complex dynamic systems in engineering, the input-output feedback linearization method based on differential geometry theory provides quite universal methods for these high-dimensional complex systems [31-34]. In order to make the designed controllers have strong robustness, the input-output feedback linearization method is often combined with sliding mode variable structure control. However, the input-output feedback linearizationbased sliding mode control methods in [35-37] often use an exponential reaching law with discontinuous terms; then, the chattering problem still widely exists in these control methods. As another variable structure control method, synergetic control can achieve similar control effect with sliding mode control on the basis of overcoming chattering [38]. Therefore, it is of great significance to propose the input-output feedback linearization-based synergetic control method for suppressing the chaotic oscillation in the complex power system.

Based on the above discussion, the dynamic models of the current source converter-based STATCOM device and energy storage device in [28-30] are introduced for the three-bus power system, and a complex controlled power system dynamic model is constructed. In order to control chaotic oscillation in the system, the whole nonlinear system is linearized step by step, and a fast synergetic control is proposed for linear systems. Since the traditional synergetic control method has the defect of slow convergence speed, we improve the traditional method by adding an exponential term to the dynamic equations satisfied by macrovariables, thus effectively improving the speed of the system state reaching the invariant manifold. The main contributions of the paper are as follows: (1) a complex coupling dynamic model composed of the power system model, energy storage device model, and current source converter-based STATCOM model is established for chaos control in a three-bus power system; (2) compared with the widely used inputoutput feedback linearization-based sliding mode control, the proposed input-output feedback linearization-based synergetic control completely avoids the chattering problem; (3) compared with traditional synergetic control, the proposed fast synergetic control improves the rapidity of control by adding an exponential term to the dynamic equation satisfied by the macrovariables of traditional synergetic control. As the system state is far away from the invariant manifold, the exponential term grows exponentially, which significantly accelerates the speed of the system state reaching the invariant manifold and improves the rapidity of traditional synergetic control.

The rest of the paper is organized as follows. In Section 2, the coupled dynamic model including the power system model, energy storage device model, and STATCOM device model is constructed, and chaotic dynamic behavior in the power system is analyzed. In Section 3, the design process of the fast synergetic controller based on input-output feedback linearization is presented. In Section 4, the effectiveness and superiority of the proposed method are verified by numerical simulation. Finally, the conclusion is given in Section 5.

\section{Power System Model and Dynamic Analysis}

The power system studied is a three-bus power system. The generator bus of the power system is connected to the energy storage device, and the load bus is connected to the energy storage device and the current source converter-based STATCOM device. The circuit diagram of the controlled power system formed after connecting these control devices is shown in Figure 1.

The coupling dynamic model of the power system in Figure 1 is established as follows: 


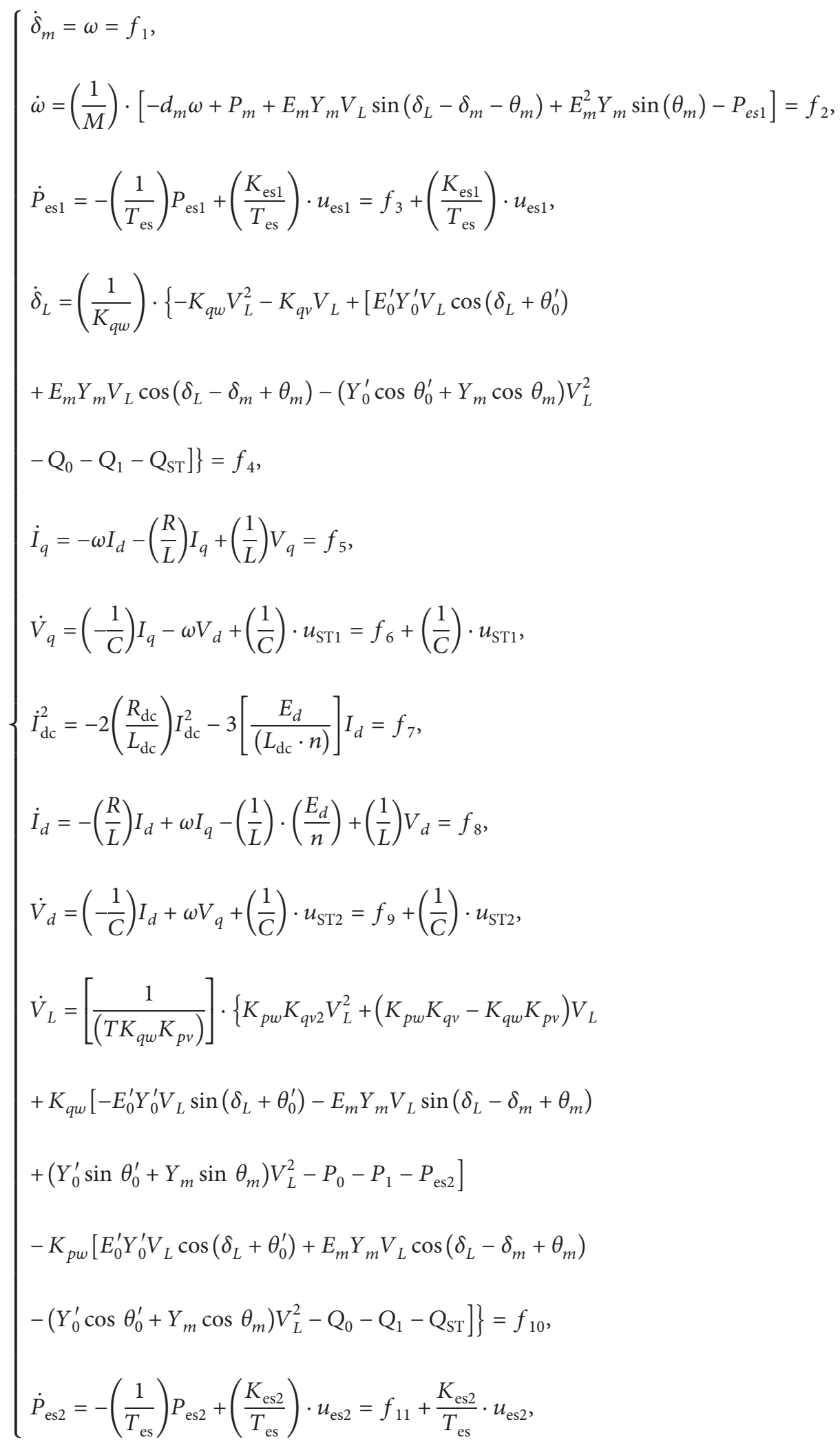

where $\delta_{m}$ and $\omega$ represent the generator power angle and frequency deviation; $\delta_{L}$ and $V_{L}$ represent the phase angle and amplitude of load bus voltage; $P_{\mathrm{es} 1}$ and $P_{\mathrm{es} 2}$ indicate the active power absorbed by two energy storage devices from the generator bus and load bus; $I_{d}$ and $I_{q}$ denote the $d$-axis and $q$-axis components of the AC side current of the 


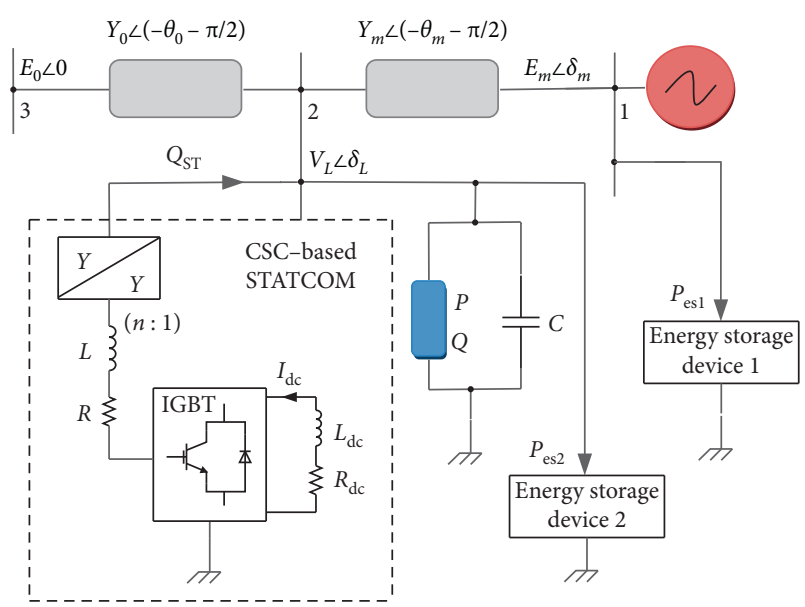

FIGURE 1: Wiring diagram of the controlled power system.

STATCOM; $V_{d}$ and $V_{q}$ represent the $d$-axis and $q$-axis components of the AC side voltage; $I_{\mathrm{dc}}^{2}$ is the square of its DC side current; and $Q_{\mathrm{ST}}=-\left(3 E_{d} I_{q}\right) / 2$ represents the reactive power injected into the load bus by the STATCOM. The eleven variables mentioned above are state variables of the controlled power system. $u_{\text {es } 1}$ and $u_{\text {es } 2}$ represent control inputs of the two energy storage devices; $u_{\mathrm{ST} 1}$ and $u_{\text {ST2 }}$ represent control inputs of the STATCOM. These four variables are input variables of the controlled power system.

Values of constant parameters in system (1) are listed as follows: $E_{m}=1.05, Y_{m}=5.0, \theta_{m}=0, E_{0}^{\prime}=20, Y_{0}^{\prime}=0.1665$, $\theta_{0}^{\prime}=0, \quad d_{m}=0.05, \quad M=0.01464, \quad K_{p w}=0.4, \quad K_{q v 2}=2.1$, $K_{q w}=-0.03, \quad K_{q v}=-2.8, \quad K_{p v}=0.3, T=8.5, \quad P_{0}=0.6$, $Q_{0}=1.3, P_{1}=0, Q_{1}=2.9, P_{m}=1.102, E_{d}=1, R_{\mathrm{dc}}=0.1$, $L_{\mathrm{dc}}=0.5, n=1, R=0.01, L=0.1, C=1.5$, and $\omega=1$. The physical meanings of parameters in the four-dimensional power system model and STATCOM device model are available in $[15,28]$. Most values of power system parameters are taken from [15] except for some important bifurcation parameters $P_{m}$ and $Q_{1}$. The initial values of state variables of system (1) are taken as follows: $\delta_{m}(0)=0.29, \omega(0)=0.2$, $P_{\text {es } 1}(0)=0, \delta_{L}(0)=0.23, I_{q}(0)=0, V_{q}(0)=0, I_{\mathrm{dc}}^{2}(0)=0$, $I_{d}(0)=0, V_{d}(0)=0, V_{L}(0)=0.8$, and $P_{\text {es } 2}(0)=0$. All the aforementioned values are in per unit except for angles, which are in degrees. Then, system (1) becomes a deterministic system with specific parameters and initial values. The first, second, fourth, and tenth equations of system (1) are considered, we let $P_{\mathrm{es} 1}=0, P_{\mathrm{es} 2}=0$, and $Q_{\mathrm{ST}}=0$, and then the dynamic system composed of these four equations is the original four-dimensional power system model. Under the given system parameters and initial values, chaotic oscillation occurs, and the strange attractor in the system is shown in Figure 2.

\section{Chaos Controller Design}

In order to control chaotic oscillation in the power system, it is necessary to design a chaos controller for controlled power system (1). The control objective of system (1) is to restore the power system to the synchronous operation state, control its bus voltage to the rated value, and maintain the DC side current of the STATCOM device to its reference value. The specific control objectives of system (1) are set as follows: $\delta_{m(\mathrm{ref})}=0, \delta_{L(\mathrm{ref})}=0, I_{\mathrm{dc}(\mathrm{ref})}^{2}=1$, and $V_{L(\mathrm{ref})}=1$. The system output functions $y_{1}, z_{1}, w_{1}$, and $m_{1}$ (i.e., output variables) are expressed as follows:

$$
\left\{\begin{array}{l}
y_{1}=L_{1} \delta_{m}, \\
z_{1}=L_{2} \delta_{L}, \\
w_{1}=L_{3}\left(I_{\mathrm{dc}}^{2}-1\right), \\
m_{1}=L_{4}\left(V_{L}-1\right),
\end{array}\right.
$$

where $L_{1}>0, L_{2}>0, L_{3}>0$, and $L_{4}>0$.

For convenience of expression, system (1) is written as a standard form of the multi-input multioutput nonlinear system as follows:

$$
\left\{\begin{array}{l}
\dot{x}=f(\mathbf{x})+g_{\mathrm{es} 1}(\mathbf{x}) u_{\mathrm{es} 1}+g_{\mathrm{ST} 1}(\mathbf{x}) u_{\mathrm{ST} 1} \\
+g_{\mathrm{ST} 2}(\mathbf{x}) u_{\mathrm{ST} 2}+g_{\mathrm{es} 2}(\mathbf{x}) u_{\mathrm{es} 2}, \\
y_{1}=h_{1}(\mathbf{x})=L_{1} \delta_{m} \\
z_{1}=h_{2}(\mathbf{x})=L_{2} \delta_{L} \\
w_{1}=h_{3}(\mathbf{x})=L_{3}\left(I_{\mathrm{dc}}^{2}-1\right), \\
m_{1}=h_{4}(x)=L_{4}\left(V_{L}-1\right),
\end{array}\right.
$$

where

$$
\left\{\begin{array}{l}
\mathbf{x}=\left[\delta_{m}, \omega, P_{\mathrm{es} 1}, \delta_{\mathrm{L}}, I_{q}, V_{q}, I_{\mathrm{dc}}^{2}, I_{d}, V_{d}, V_{L}, P_{\mathrm{es} 2}\right]^{T}, \\
f(\mathbf{x})=\left[f_{1}, f_{2}, f_{3}, f_{4}, f_{5}, f_{6}, f_{7}, f_{8}, f_{9}, f_{10}, f_{11}\right]^{T}, \\
g_{\mathrm{es} 1}(\mathbf{x})=\left[0,0,\left(\frac{K_{\mathrm{es} 2}}{T_{\mathrm{es}}}\right), 0,0,0,0,0,0,0,0\right]^{T}, \\
g_{\mathrm{ST} 1}(\mathbf{x})=\left[0,0,0,0,0,\left(\frac{1}{C}\right), 0,0,0,0,0\right]^{T}, \\
g_{\mathrm{ST} 2}(\mathbf{x})=\left[0,0,0,0,0,0,0,0,\left(\frac{1}{C}\right), 0,0\right]^{T}, \\
g_{\mathrm{es} 2}(\mathbf{x})=\left[0,0,0,0,0,0,0,0,0,0,\left(\frac{K_{\mathrm{es} 2}}{T_{\mathrm{es}}}\right)\right]^{T} .
\end{array}\right.
$$

3.1. Input-Output Feedback Linearization Process. The core idea of input-output feedback linearization is to transform the control problem of a complex nonlinear system into the control of linear systems through nonlinear coordinate transformation. The core step is to derive the output function step by step until at least one control input appears in the linear system in the form of explicit function [39]. The following are the derivations of the output functions: 


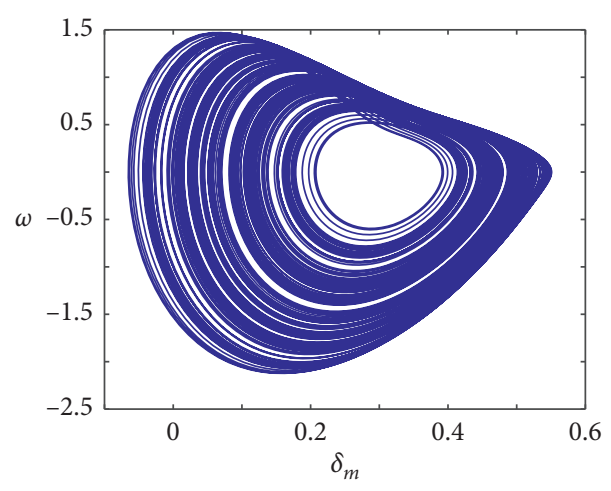

(a)

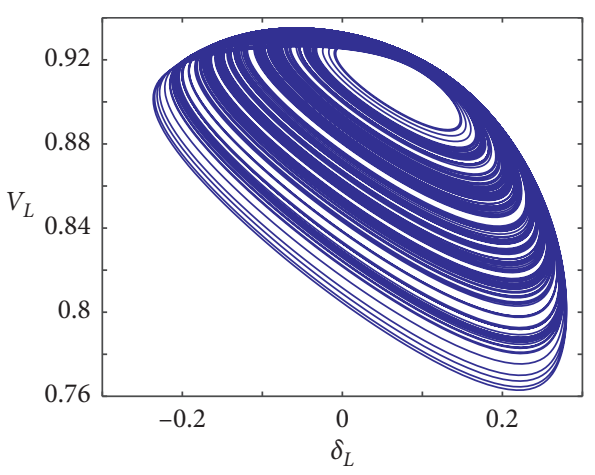

(b)

FIgURE 2: Strange attractor in the power system.

(1) The derivatives of the first output function $y_{1}$ in (2) are obtained step by step. The first derivative of $y_{1}$ is

$$
\begin{aligned}
\dot{y}_{1}= & \frac{\partial h_{1}(\mathbf{x})}{\partial \mathbf{x}}\left[f(\mathbf{x})+g_{\mathrm{es} 1}(\mathbf{x}) u_{\mathrm{es} 1}+g_{\mathrm{ST} 1}(\mathbf{x}) u_{\mathrm{ST} 1}\right. \\
& \left.+g_{\mathrm{ST} 2}(\mathbf{x}) u_{\mathrm{ST} 2}+g_{\mathrm{es} 2}(\mathbf{x}) u_{\mathrm{es} 2}\right], \\
= & L_{f} h_{1}(\mathbf{x})+L_{g_{\mathrm{es} 1}} h_{1}(\mathbf{x}) \cdot u_{\mathrm{es} 1}+L_{g_{\mathrm{ST} 1}} h_{1}(\mathbf{x}) \cdot u_{\mathrm{ST} 1} \\
& +L_{g_{\mathrm{ST} 2}} h_{1}(\mathbf{x}) \cdot u_{\mathrm{ST} 2}+L_{g_{\mathrm{es} 2}} h_{1}(\mathbf{x}) \cdot u_{\mathrm{es} 2} .
\end{aligned}
$$

Since

$$
\left\{\begin{array}{l}
L_{g_{\mathrm{es} 1}} h_{1}(\mathbf{x})=0, \\
L_{g_{\mathrm{ST} 1}} h_{1}(\mathbf{x})=0, \\
L_{g_{\mathrm{ST} 2}} h_{1}(\mathbf{x})=0, \\
L_{g_{\mathrm{es} 2}} h_{1}(\mathbf{x})=0,
\end{array}\right.
$$

system (5) becomes

$$
\dot{y}_{1}=L_{f} h_{1}(\mathbf{x})=L_{1} \cdot f_{1}=L_{1} \cdot \omega .
$$

There is no control input in system (7); then, continue to calculate the second derivative of $y_{1}$ as follows:

$$
\begin{aligned}
\ddot{y}_{1}= & \frac{\partial\left[L_{f} h_{1}(\mathbf{x})\right]}{\partial \mathbf{x}}\left[f(\mathbf{x})+g_{\mathrm{es} 1}(\mathbf{x}) u_{\mathrm{es} 1}+g_{\mathrm{ST} 1}(\mathbf{x}) u_{\mathrm{ST} 1}\right. \\
& \left.+g_{\mathrm{ST} 2}(\mathbf{x}) u_{\mathrm{ST} 2}+g_{\mathrm{es} 2}(\mathbf{x}) u_{\mathrm{es} 2}\right], \\
= & L_{f}^{2} h_{1}(\mathbf{x})+L_{g_{\mathrm{es} 1}} L_{f} h_{1}(\mathbf{x}) \cdot u_{\mathrm{es} 1}+L_{g_{\mathrm{ST} 1}} L_{f} h_{1}(\mathbf{x}) \cdot u_{\mathrm{ST} 1} \\
& +L_{g_{\mathrm{ST} 2}} L_{f} h_{1}(\mathbf{x}) \cdot u_{\mathrm{ST} 2}+L_{g_{\mathrm{es} 2}} L_{f} h_{1}(\mathbf{x}) \cdot u_{\mathrm{es} 2} .
\end{aligned}
$$

$$
\left\{\begin{array}{l}
L_{g_{\mathrm{es} 1}} L_{f} h_{1}(\mathbf{x})=0, \\
L_{g_{\mathrm{ST} 1}} L_{f} h_{1}(\mathbf{x})=0, \\
L_{g_{\mathrm{ST} 2}} L_{f} h_{1}(\mathbf{x})=0, \\
L_{g_{\mathrm{es} 2}} L_{f} h_{1}(\mathbf{x})=0,
\end{array}\right.
$$

system (8) becomes

$$
\ddot{y}_{1}=L_{f}^{2} h_{1}(\mathbf{x})=L_{1} \cdot f_{2} .
$$

There is still no control input in system (10) in the form of the explicit function; we continue to calculate the third derivative of $y_{1}$ as follows:

$$
\begin{aligned}
\dddot{y}_{1}= & \frac{\partial\left[L_{f}^{2} h_{1}(\mathbf{x})\right]}{\partial \mathbf{x}}\left[f(\mathbf{x})+g_{\mathrm{es} 1}(\mathbf{x}) u_{\mathrm{es} 1}+g_{\mathrm{ST} 1}(\mathbf{x}) u_{\mathrm{ST} 1}\right. \\
& \left.+g_{\mathrm{ST} 2}(\mathbf{x}) u_{\mathrm{ST} 2}+g_{\mathrm{es} 2}(\mathbf{x}) u_{\mathrm{es} 2}\right], \\
= & L_{f}^{3} h_{1}(\mathbf{x})+L_{g_{\mathrm{es} 1}} L_{f}^{2} h_{1}(\mathbf{x}) \cdot u_{\mathrm{es} 1}+L_{g_{\mathrm{ST} 1}} L_{f}^{2} h_{1}(\mathbf{x}) \cdot u_{\mathrm{ST} 1} \\
& +L_{g_{\mathrm{ST} 2}} L_{f}^{2} h_{1}(\mathbf{x}) \cdot u_{\mathrm{ST} 2}+L_{g_{\mathrm{es} 2}} L_{f}^{2} h_{1}(\mathbf{x}) \cdot u_{\mathrm{e} 22} .
\end{aligned}
$$

Since

$$
\left\{\begin{array}{l}
L_{g_{\mathrm{es} 1}} L_{f}^{2} h_{1}(\mathbf{x})=-L_{1} \frac{K_{\mathrm{es} 1}}{\left(M \cdot T_{\mathrm{es}}\right)}, \\
L_{g_{\mathrm{ST} 1}} L_{f}^{2} h_{1}(\mathbf{x})=0 \\
L_{g_{\mathrm{ST} 2}} L_{f}^{2} h_{1}(\mathbf{x})=0 \\
L_{g_{\mathrm{es} 2}} L_{f}^{2} h_{1}(\mathbf{x})=0
\end{array}\right.
$$

system (11) becomes

$$
\dddot{y}_{1}=L_{f}^{3} h_{1}(\mathbf{x})+L_{g_{\mathrm{es} 1}} L_{f}^{2} h_{1}(\mathbf{x}) \cdot u_{\mathrm{es} 1} .
$$


Since $L_{g_{\text {es } 1}} L_{f}^{2} h_{1}(\mathbf{x}) \neq 0$, the control input $u_{\text {es } 1}$ will exist in system (13) in the form of an explicit function, and system (13) is equivalent to a linear system as follows:

$$
\left\{\begin{array}{l}
\dot{y}_{1}=y_{2}, \\
\dot{y}_{2}=y_{3}, \\
\dot{y}_{3}=v_{1},
\end{array}\right.
$$

where $v_{1}=L_{f}^{3} h_{1}(\mathbf{x})+L_{g_{\mathrm{es} 1}} L_{f}^{2} h_{1}(\mathbf{x}) \cdot u_{\mathrm{es} 1}$, representing the virtual control input of system (14).

In this way, the linear relationship between the virtual control input $v_{1}$ and the output function $y_{1}$ is established, and the controller design problem of the control input $u_{\text {es } 1}$ in nonlinear system (1) is transformed into the design process of the control input $v_{1}$ in linear system (14). The transformation relationship between $u_{\text {es } 1}$ and $v_{1}$ is

$$
u_{\mathrm{es} 1}=\frac{-L_{f}^{3} h_{1}(\mathbf{x})+v_{1}}{L_{g_{\mathrm{es} 1}} L_{f}^{2} h_{1}(\mathbf{x})}
$$

(2) The derivatives of the second output function $z_{1}$ in (2) are obtained step by step until at least one control input appears in the system in the form of the explicit function. First, the first derivative of $z_{1}$ is obtained as follows:

$$
\begin{aligned}
\dot{z}_{1}= & \frac{\partial h_{2}(\mathbf{x})}{\partial \mathbf{x}}\left[f(\mathbf{x})+g_{\mathrm{es} 1}(\mathbf{x}) u_{\mathrm{es} 1}+g_{\mathrm{ST} 1}(\mathbf{x}) u_{\mathrm{ST} 1}\right. \\
& \left.+g_{\mathrm{ST} 2}(\mathbf{x}) u_{\mathrm{ST} 2}+g_{\mathrm{es} 2}(\mathbf{x}) u_{\mathrm{es} 2}\right] \\
= & L_{f} h_{2}(\mathbf{x})+L_{g_{\mathrm{es} 1}} h_{2}(\mathbf{x}) \cdot u_{\mathrm{es} 1}+L_{g_{\mathrm{ST} 1}} h_{2}(\mathbf{x}) \cdot u_{\mathrm{ST} 1} \\
& +L_{g_{\mathrm{ST} 2}} h_{2}(\mathbf{x}) \cdot u_{\mathrm{ST} 2}+L_{g_{\mathrm{es} 2}} h_{2}(\mathbf{x}) \cdot u_{\mathrm{es} 2} .
\end{aligned}
$$

In (16), since

$$
\left\{\begin{array}{l}
L_{g_{\mathrm{es} 1}} h_{2}(\mathbf{x})=0, \\
L_{g_{\mathrm{ST} 1}} h_{2}(\mathbf{x})=0, \\
L_{g_{\mathrm{ST} 2}} h_{2}(\mathbf{x})=0, \\
L_{g_{\mathrm{es} 2}} h_{2}(\mathbf{x})=0,
\end{array}\right.
$$

system (16) becomes

$$
\dot{z}_{1}=L_{f} h_{2}(\mathbf{x})=L_{2} \cdot f_{4} .
$$

Since there is no control input in system (18) in the form of an explicit function, we continue to calculate the second derivative of $z_{1}$ as follows:

$$
\begin{aligned}
\ddot{z}_{1}= & \frac{\partial\left[L_{f} h_{2}(\mathbf{x})\right]}{\partial \mathbf{x}}\left[f(\mathbf{x})+g_{\mathrm{es} 1}(\mathbf{x}) u_{\mathrm{es} 1}+g_{\mathrm{ST} 1}(\mathbf{x}) u_{\mathrm{ST} 1}\right. \\
& \left.+g_{\mathrm{ST} 2}(\mathbf{x}) u_{\mathrm{ST} 2}+g_{\mathrm{es} 2}(\mathbf{x}) u_{\mathrm{es} 2}\right] . \\
= & L_{f}^{2} h_{2}(\mathbf{x})+L_{g_{\mathrm{es} 1}} L_{f} h_{2}(\mathbf{x}) \cdot u_{\mathrm{es} 1}+L_{g_{\mathrm{ST} 1}} L_{f} h_{2}(\mathbf{x}) \cdot u_{\mathrm{ST} 1} \\
& +L_{g_{\mathrm{ST} 2}} L_{f} h_{2}(\mathbf{x}) \cdot u_{\mathrm{ST} 2}+L_{g_{\mathrm{es} 2}} L_{f} h_{2}(\mathbf{x}) \cdot u_{\mathrm{es} 2} .
\end{aligned}
$$

In (19), since

$$
\left\{\begin{array}{l}
L_{g_{\mathrm{es} 1}} L_{f} h_{2}(\mathbf{x})=0, \\
L_{g_{\mathrm{ST} 1}} L_{f} h_{2}(\mathbf{x})=0, \\
L_{g_{\mathrm{ST} 2}} L_{f} h_{2}(\mathbf{x})=0, \\
L_{g_{\mathrm{es} 2}} L_{f} h_{2}(\mathbf{x})=0,
\end{array}\right.
$$

system (19) becomes

$$
\ddot{z}_{1}=L_{f}^{2} h_{2}(\mathbf{x})
$$

Since there is no control input in (21) in the form of an explicit function, the third derivative of $z_{1}$ is obtained as follows:

$$
\begin{aligned}
\dddot{z}_{1}= & \frac{\partial\left[L_{f}^{2} h_{2}(\mathbf{x})\right]}{\partial \mathbf{x}}\left[f(\mathbf{x})+g_{\mathrm{es} 1}(\mathbf{x}) u_{\mathrm{es} 1}\right. \\
& \left.+g_{\mathrm{ST} 1}(\mathbf{x}) u_{\mathrm{ST} 1}+g_{\mathrm{ST} 2}(\mathbf{x}) u_{\mathrm{ST} 2}+g_{\mathrm{es} 2}(\mathbf{x}) u_{\mathrm{es} 2}\right], \\
= & L_{f}^{3} h_{2}(\mathbf{x})+L_{g_{\mathrm{es} 1}} L_{f}^{2} h_{2}(\mathbf{x}) \cdot u_{\mathrm{es} 1}+L_{g_{\mathrm{ST} 1}} L_{f}^{2} h_{2}(\mathbf{x}) \cdot u_{\mathrm{ST} 1} \\
& +L_{g_{\mathrm{ST} 2}} L_{f}^{2} h_{2}(\mathbf{x}) \cdot u_{\mathrm{ST} 2}+L_{g_{\mathrm{es} 2}} L_{f}^{2} h_{2}(\mathbf{x}) \cdot u_{\mathrm{es} 2} .
\end{aligned}
$$

In (22),

$$
\left\{\begin{array}{l}
L_{g_{\mathrm{es} 1}} L_{f}^{2} h_{2}(\mathbf{x})=0, \\
L_{g_{\mathrm{ST} 2}} L_{f}^{2} h_{2}(\mathbf{x})=0, \\
L_{g_{\mathrm{es} 2}} L_{f}^{2} h_{2}(\mathbf{x})=0 \\
L_{g_{\mathrm{ST} 1}} L_{f}^{2} h_{2}(\mathbf{x})=\frac{\partial\left[L_{f}^{2} h_{2}(\mathbf{x})\right]}{\partial V_{q}} \cdot \frac{1}{C}=L_{2} \cdot\left(\frac{1}{K_{q w}}\right) \cdot\left(\frac{3 E_{d}}{2}\right) \cdot\left(\frac{1}{L C}\right) .
\end{array}\right.
$$


In this way, system (21) is transformed into

$$
\dddot{z}_{1}=L_{f}^{3} h_{2}(\mathbf{x})+L_{g_{\mathrm{ST} 1}} L_{f}^{2} h_{2}(\mathbf{x}) \cdot u_{\mathrm{ST} 1} .
$$

Similarly, since $L_{g_{\mathrm{ST} 1}} L_{f}^{2} h_{2}(\mathbf{x}) \neq 0$, the control input $u_{\mathrm{ST} 1}$ in (24) appears in the form of an explicit function, while system (24) is equivalent to a linear system:

$$
\left\{\begin{array}{l}
\dot{z}_{1}=z_{2}, \\
\dot{z}_{2}=z_{3}, \\
\dot{z}_{3}=v_{2},
\end{array}\right.
$$

where $\quad v_{2}=L_{f}^{3} h_{2}(\mathbf{x})+L_{g_{\mathrm{ST} 1}} L_{f}^{2} h_{2}(\mathbf{x}) \cdot u_{\mathrm{ST} 1}$, representing the virtual control input of system (25).

In this way, the controller design of $u_{\mathrm{ST} 1}$ for nonlinear system (1) is transformed into the design process of the control input $v_{2}$ for linear system (25). The transformation relationship between $u_{\mathrm{ST} 1}$ and $v_{2}$ is as follows:

$$
u_{\mathrm{ST} 1}=\frac{-L_{f}^{3} h_{2}(\mathbf{x})+v_{2}}{L_{g_{\mathrm{ST} 1}} L_{f}^{2} h_{2}(\mathbf{x})} .
$$

(3) The derivatives of the third output function $w_{1}$ in (2) are obtained step by step until at least one control input appears in the system in the form of an explicit function, and the first derivative of $w_{1}$ is obtained as follows:

$$
\begin{aligned}
\dot{w}_{1}= & \frac{\partial h_{3}(\mathbf{x})}{\partial \mathbf{x}}\left[f(\mathbf{x})+g_{\mathrm{es} 1}(\mathbf{x}) u_{\mathrm{es} 1}+g_{\mathrm{ST} 1}(\mathbf{x}) u_{\mathrm{ST} 1}\right. \\
& \left.+g_{\mathrm{ST} 2}(\mathbf{x}) u_{\mathrm{ST} 2}+g_{\mathrm{es} 2}(\mathbf{x}) u_{\mathrm{es} 2}\right] . \\
= & L_{f} h_{3}(\mathbf{x})+L_{g_{\mathrm{es} 1}} h_{3}(\mathbf{x}) \cdot u_{\mathrm{es} 1}+L_{g_{\mathrm{ST} 1}} h_{3}(\mathbf{x}) \cdot u_{\mathrm{ST} 1} \\
& +L_{g_{\mathrm{ST} 2}} h_{3}(\mathbf{x}) \cdot u_{\mathrm{ST} 2}+L_{g_{\mathrm{es} 2}} h_{3}(\mathbf{x}) \cdot u_{\mathrm{es} 2} .
\end{aligned}
$$

$$
\left\{\begin{array}{l}
L_{g_{\mathrm{es} 1}} h_{3}(\mathbf{x})=0, \\
L_{g_{\mathrm{ST} 1}} h_{3}(\mathbf{x})=0, \\
L_{g_{\mathrm{ST} 2}} h_{3}(\mathbf{x})=0, \\
L_{g_{\mathrm{es} 2}} h_{3}(\mathbf{x})=0,
\end{array}\right.
$$

the system can be changed into

$$
\dot{w}_{1}=L_{f} h_{3}(\mathbf{x})=L_{3} \cdot f_{7} .
$$

Since there is no control input in system (27) in the form of an explicit function, we continue to calculate the second derivative of $w_{1}$ as follows:

$$
\begin{aligned}
w_{1}= & \frac{\partial\left[L_{f} h_{3}(\mathbf{x})\right]}{\partial \mathbf{x}}\left[f(\mathbf{x})+g_{\mathrm{es} 1}(\mathbf{x}) u_{\mathrm{es} 1}+g_{\mathrm{ST} 1}(\mathbf{x}) u_{\mathrm{ST} 1}\right. \\
& \left.+g_{\mathrm{ST} 2}(\mathbf{x}) u_{\mathrm{ST} 2}+g_{\mathrm{es} 2}(\mathbf{x}) u_{\mathrm{es} 2}\right] \\
= & L_{f}^{2} h_{3}(\mathbf{x})+L_{g_{\mathrm{es} 1}} L_{f} h_{3}(\mathbf{x}) \cdot u_{\mathrm{es} 1}+L_{g_{\mathrm{ST} 1}} L_{f} h_{3}(\mathbf{x}) \\
& \cdot u_{\mathrm{ST} 1}+L_{g_{\mathrm{ST} 2}} L_{f} h_{3}(\mathbf{x}) \cdot u_{\mathrm{ST} 2}+L_{g_{\mathrm{es} 2}} L_{f} h_{3}(\mathbf{x}) \cdot u_{\mathrm{es} 2} .
\end{aligned}
$$

Since

$$
\left\{\begin{array}{l}
L_{g_{\mathrm{es} 1}} L_{f} h_{3}(\mathbf{x})=0, \\
L_{g_{\mathrm{ST} 1}} L_{f} h_{3}(\mathbf{x})=0, \\
L_{g_{\mathrm{ST} 2}} L_{f} h_{3}(\mathbf{x})=0, \\
L_{g_{\mathrm{es} 2}} L_{f} h_{3}(\mathbf{x})=0,
\end{array}\right.
$$

system (30) becomes

$$
\ddot{w}_{1}=L_{f}^{2} h_{3}(\mathbf{x}) .
$$

Since there is no control input in (32) that appears in the form of an explicit function, we continue to calculate the third derivative of $w_{1}$ as follows:

In (27), since

$$
\begin{aligned}
\dddot{w}_{1}= & \frac{\partial\left[L_{f}^{2} h_{3}(\mathbf{x})\right]}{\partial \mathbf{x}}\left[f(\mathbf{x})+g_{\mathrm{es} 1}(\mathbf{x}) u_{\mathrm{es} 1}\right. \\
& \left.+g_{\mathrm{ST} 1}(\mathbf{x}) u_{\mathrm{ST} 1}+g_{\mathrm{ST} 2}(\mathbf{x}) u_{\mathrm{ST} 2}+g_{\mathrm{es} 2}(\mathbf{x}) u_{\mathrm{es} 2}\right] \\
= & L_{f}^{3} h_{3}(\mathbf{x})+L_{g_{\mathrm{es} 1}} L_{f}^{2} h_{3}(\mathbf{x}) \cdot u_{\mathrm{es} 1} \\
& +L_{g_{\mathrm{ST} 1}} L_{f}^{2} h_{3}(\mathbf{x}) \cdot u_{\mathrm{ST} 1}+L_{g_{\mathrm{ST} 2}} L_{f}^{2} h_{3}(\mathbf{x}) \cdot u_{\mathrm{ST} 2}+L_{g_{\mathrm{es} 2}} L_{f}^{2} h_{3}(\mathbf{x}) \cdot u_{\mathrm{es} 2} .
\end{aligned}
$$


In (33), since

$$
\left\{\begin{array}{l}
L_{g_{\mathrm{es} 1}} L_{f}^{2} h_{3}(\mathbf{x})=0 \\
L_{g_{\mathrm{ST} 1}} L_{f}^{2} h_{3}(\mathbf{x})=0 \\
L_{g_{\mathrm{es} 2}} L_{f}^{2} h_{3}(\mathbf{x})=0 \\
L_{g_{\mathrm{ST} 2}} L_{f}^{2} h_{3}(\mathbf{x})=\frac{\partial\left[L_{f}^{2} h_{3}(\mathbf{x})\right]}{\partial V_{d}} \cdot \frac{1}{C}=L_{3} \cdot\left(\frac{-3 E_{d}}{L_{\mathrm{dc}} \cdot n}\right) \cdot\left(\frac{1}{L C}\right),
\end{array}\right.
$$

the system becomes

$$
\dddot{w}_{1}=L_{f}^{3} h_{3}(\mathbf{x})+L_{g_{\mathrm{ST} 2}} L_{f}^{2} h_{3}(\mathbf{x}) \cdot u_{\mathrm{ST} 2} .
$$

In (35), since $L_{g_{\mathrm{ST} 2}} L_{f}^{2} h_{3}(\mathbf{x}) \neq 0, u_{\mathrm{ST} 2}$ appears in system (33) in the form of an explicit function, and system (35) is equivalent to a linear system as follows:

$$
\left\{\begin{array}{l}
\dot{w}_{1}=w_{2}, \\
\dot{w}_{2}=w_{3}, \\
\dot{w}_{3}=v_{3},
\end{array}\right.
$$

where $\quad v_{3}=L_{f}^{3} h_{3}(\mathbf{x})+L_{g_{\mathrm{ST} 2}} L_{f}^{2} h_{3}(\mathbf{x}) \cdot u_{\mathrm{ST} 2}$, representing the virtual control input of system (36).

In this way, the controller design of $u_{\mathrm{ST} 2}$ for system (1) is transformed into the design process of control input $v_{3}$ for linear system (36). That is, as long as $u_{\mathrm{ST} 2}$ is designed for system (36) to make the whole linear system stable to the origin, the third output of system (1) will be controlled to the target. The transformation relationship between $u_{\mathrm{ST} 2}$ and $v_{3}$ is as follows:

$$
u_{\mathrm{ST} 2}=\frac{-L_{f}^{3} h_{3}(\mathbf{x})+v_{3}}{L_{g_{\mathrm{ST} 2}} L_{f}^{2} h_{3}(\mathbf{x})} .
$$

(4) The derivatives of the fourth output function $m_{1}$ in (2) are obtained step by step until at least one control input appears in the system in the form of an explicit function, and the first derivative of $m_{1}$ is obtained as follows:

$$
\begin{aligned}
\dot{m}_{1}= & \frac{\partial h_{4}(\mathbf{x})}{\partial \mathbf{x}}\left[f(\mathbf{x})+g_{\mathrm{es} 1}(\mathbf{x}) u_{\mathrm{es} 1}+g_{\mathrm{ST} 1}(\mathbf{x}) u_{\mathrm{ST} 1}\right. \\
& \left.+g_{\mathrm{ST} 2}(\mathbf{x}) u_{\mathrm{ST} 2}+g_{\mathrm{es} 2}(\mathbf{x}) u_{\mathrm{es} 2}\right], \\
= & L_{f} h_{4}(\mathbf{x})+L_{g_{\mathrm{es} 1}} h_{4}(\mathbf{x}) \cdot u_{\mathrm{es} 1}+L_{g_{\mathrm{ST} 1}} h_{4}(\mathbf{x}) \cdot u_{\mathrm{ST} 1} \\
& +L_{g_{\mathrm{ST} 2}} h_{4}(\mathbf{x}) \cdot u_{\mathrm{ST} 2}+L_{g_{\mathrm{es} 2}} h_{4}(\mathbf{x}) \cdot u_{\mathrm{es} 2} .
\end{aligned}
$$

In (36), since

$$
\left\{\begin{array}{l}
L_{g_{\mathrm{es} 1}} h_{4}(\mathbf{x})=0, \\
L_{g_{\mathrm{ST} 1}} h_{4}(\mathbf{x})=0, \\
L_{g_{\mathrm{ST} 2}} h_{4}(\mathbf{x})=0, \\
L_{g_{\mathrm{es} 2}} h_{4}(\mathbf{x})=0,
\end{array}\right.
$$

system (38) can be changed into

$$
\dot{m}_{1}=L_{f} h_{4}(\mathbf{x})=L_{4} \cdot f_{10} .
$$

Since there is no control input in system (40) in the form of an explicit function, we continue to calculate the second derivative of $m_{1}$ as follows:

$$
\begin{aligned}
\ddot{m}_{1}= & \frac{\partial\left[L_{f} h_{4}(\mathbf{x})\right]}{\partial \mathbf{x}}\left[f(\mathbf{x})+g_{\mathrm{es} 1}(\mathbf{x}) u_{\mathrm{es} 1}+g_{\mathrm{ST} 1}(\mathbf{x}) u_{\mathrm{ST} 1}\right. \\
& \left.+g_{\mathrm{ST} 2}(\mathbf{x}) u_{\mathrm{ST} 2}+g_{\mathrm{es} 2}(\mathbf{x}) u_{\mathrm{es} 2}\right] \\
= & L_{f}^{2} h_{4}(\mathbf{x})+L_{g_{\mathrm{es} 1}} L_{f} h_{4}(\mathbf{x}) \cdot u_{\mathrm{es} 1}+L_{g_{\mathrm{ST} 1}} L_{f} h_{4}(\mathbf{x}) \cdot u_{\mathrm{ST} 1} \\
& +L_{g_{\mathrm{ST} 2}} L_{f} h_{4}(\mathbf{x}) \cdot u_{\mathrm{ST} 2}+L_{g_{\mathrm{es} 2}} L_{f} h_{4}(\mathbf{x}) \cdot u_{\mathrm{es} 2} .
\end{aligned}
$$

In (41), since

$$
\left\{\begin{array}{l}
L_{g_{\mathrm{es} 1}} L_{f} h_{4}(\mathbf{x})=0, \\
L_{g_{\mathrm{ST} 1}} L_{f} h_{4}(\mathbf{x})=0, \\
L_{g_{\mathrm{ST} 2}} L_{f} h_{4}(\mathbf{x})=0 \\
L_{g_{\mathrm{e} 2}} L_{f} h_{4}(\mathbf{x})=\frac{-L_{4} K_{\mathrm{es} 2}}{\left(T \cdot K_{p v} \cdot T_{\mathrm{es}}\right)}
\end{array}\right.
$$

the system becomes

$$
\ddot{m}_{1}=L_{f}^{2} h_{4}(\mathbf{x})+L_{g_{\mathrm{es} 2}} L_{f} h_{4}(\mathbf{x}) \cdot u_{\mathrm{es} 2} .
$$

In (43), since $L_{g_{\mathrm{es} 2}} L_{f} h_{4}(\mathbf{x}) \neq 0, u_{\mathrm{es} 2}$ appears in system (43) in the form of an explicit function, and system (43) is equivalent to a linear system:

$$
\left\{\begin{array}{l}
\dot{m}_{1}=m_{2}, \\
\dot{m}_{2}=v_{4},
\end{array}\right.
$$

where $v_{4}=L_{f}^{2} h_{4}(\mathbf{x})+L_{g_{\mathrm{es} 2}} L_{f} h_{4}(\mathbf{x}) \cdot u_{\mathrm{es} 2}$, representing the virtual control input of system (44).

In this way, the design of controller $u_{\mathrm{es} 2}$ for nonlinear system (1) is transformed into the controller design problem for linear system (44). The transformation relationship between $u_{\text {es } 2}$ and $v_{4}$ is as follows:

$$
u_{\mathrm{es} 2}=\frac{-L_{f}^{2} h_{4}(\mathbf{x})+v_{4}}{L_{g_{\mathrm{es} 2}} L_{f} h_{4}(\mathbf{x})} .
$$

By using the input-output feedback linearization method, the controller design problems of four control inputs $u_{\mathrm{es} 1}, u_{\mathrm{ST} 1}, u_{\mathrm{ST} 2}$, and $u_{\mathrm{es} 2}$ in system (1) are transformed 
into the stabilization of linear systems (14), (25), (36), and (44). If the linear systems are controlled to the origin by designing control inputs $u_{\mathrm{es} 1}, u_{\mathrm{ST} 1}, u_{\mathrm{ST} 2}$, and $u_{\mathrm{es} 2}$, then the four outputs in (2) can be restored to the origin, and the control objective of system (1) can be realized. After the above derivation, the linearization process of controlled power system (1) is completed.

3.2. Fast Synergetic Controller Design. Synergetic control inputs can be designed for linear systems (14), (25), (36), and (44) to control them to the origin. Since four control inputs need to be designed, four macrovariables are defined as follows:

$$
\left\{\begin{array}{l}
\psi_{1}=y_{3}+\alpha_{2} y_{2}+\alpha_{1} y_{1}, \\
\psi_{2}=z_{3}+\beta_{2} z_{2}+\beta_{1} z_{1}, \\
\psi_{3}=w_{3}+\gamma_{2} w_{2}+\gamma_{1} w_{1}, \\
\psi_{4}=m_{2}+\eta_{1} m_{1}
\end{array}\right.
$$

where values of parameters $\alpha_{2}, \alpha_{1}, \beta_{2}, \beta_{1}, \gamma_{2}, \gamma_{1}$, and $\eta_{1}$ should make the roots of characteristic equations $s^{2}+\alpha_{2} s+\alpha_{1}=0, s^{2}+\beta_{2} s+\beta_{1}=0, s^{2}+\gamma_{2} s+\gamma_{1}=0$, and $s+$ $\eta_{1}=0$ located in the left half plane of the complex plane.

To make the system state reach the invariant manifolds $\psi_{1}=0, \psi_{2}=0, \psi_{3}=0$, and $\psi_{4}=0$, respectively, let the dynamic equations satisfied by macrovariables be

$$
\left\{\begin{array}{c}
T_{1} \dot{\psi}_{1}+e^{\left|\psi_{1}\right|} \psi_{1}=0, \\
T_{2} \dot{\psi}_{2}+e^{\left|\psi_{2}\right|} \psi_{2}=0 \\
T_{3} \dot{\psi}_{3}+e^{\left|\psi_{3}\right|} \psi_{3}=0, \\
T_{4} \dot{\psi}_{4}+e^{\left|\psi_{4}\right|} \psi_{4}=0,
\end{array}\right.
$$

where $T_{1}>0, T_{2}>0, T_{3}>0$, and $T_{4}>0$.

Remark 1. According to the traditional synergetic control [40-45], equations in (47) should be expressed as follows:

$$
\left\{\begin{array}{l}
T_{1} \dot{\psi}_{1}+\psi_{1}=0, \\
T_{2} \dot{\psi}_{2}+\psi_{2}=0, \\
T_{3} \dot{\psi}_{3}+\psi_{3}=0, \\
T_{4} \dot{\psi}_{4}+\psi_{4}=0 .
\end{array}\right.
$$

However, since $e^{\left|\psi_{i}\right|} \geq 1(i=1,2,3,4)$ in (47), the larger $\left|\psi_{i}\right|$ is, the larger the coefficient $e^{\left|\psi_{i}\right|}$ is, and it will grow exponentially. That is to say, with the same values of $T_{i}(i=1,2,3,4)$, the farther the system state is from the invariant manifold, the faster the coefficient $e^{\left|\psi_{i}\right|}$ increases exponentially, and it significantly improves the speed of the system state reaching the invariant manifold.

Combining (46) and (47) with the last equation of (14), (25), (36), and (44), the control inputs are obtained as follows:

$$
\left\{\begin{array}{l}
u_{\mathrm{es} 1}=-\frac{1}{L_{g_{\mathrm{es} 1}} L_{f}^{2} h_{1}(\mathbf{x})}\left[L_{f}^{3} h_{1}(\mathbf{x})+\alpha_{2} y_{3}+\alpha_{1} y_{2}+\frac{e^{\left|\psi_{1}\right|} \cdot \psi_{1}}{T_{1}}\right], \\
u_{\mathrm{ST} 1}=-\frac{1}{L_{g_{\mathrm{ST} 1}} L_{f}^{2} h_{2}(\mathbf{x})}\left[L_{f}^{3} h_{2}(\mathbf{x})+\beta_{2} z_{3}+\beta_{1} z_{2}+\frac{e^{\left|\psi_{2}\right|} \cdot \psi_{2}}{T_{2}}\right], \\
u_{\mathrm{ST} 2}=-\frac{1}{L_{g_{\mathrm{ST} 2}} L_{f}^{2} h_{3}(\mathbf{x})}\left[L_{f}^{3} h_{3}(\mathbf{x})+\gamma_{2} w_{3}+\gamma_{1} w_{2}+\frac{e^{\left|\psi_{3}\right|} \cdot \psi_{3}}{T_{3}}\right], \\
u_{\mathrm{es} 2}=-\frac{1}{L_{g_{\mathrm{es} 2} L_{f} h_{4}(\mathbf{x})}}\left[L_{f}^{2} h_{4}(\mathbf{x})+\eta_{1} m_{2}+\frac{e^{\left|\psi_{4}\right|} \cdot \psi_{4}}{T_{4}}\right] .
\end{array}\right.
$$

However, due to the limitation of relative degree, control inputs (49) contain complex system functions with derivative terms $L_{f}^{3} h_{1}(\mathbf{x}), L_{f}^{3} h_{2}(\mathbf{x}), L_{f}^{3} h_{3}(\mathbf{x})$, and $L_{f}^{2} h_{4}(\mathbf{x})$. In particular, $L_{f}^{3} h_{2}(\mathbf{x})$ and $L_{f}^{2} h_{4}(\mathbf{x})$ are more complex and extremely difficult to obtain, and they also reduce the engineering practicability of controllers (49). Therefore, we assume that $L_{f}^{3} h_{1}(\mathbf{x}), L_{f}^{3} h_{2}(\mathbf{x}), L_{f}^{3} h_{3}(\mathbf{x})$, and $L_{f}^{2} h_{4}(\mathbf{x})$ satisfy assumptions (50) and redesign the controllers. 


$$
\left\{\begin{array}{l}
\left|L_{f}^{3} h_{1}(\mathbf{x})\right| \leq k_{1}\left|\tanh \left(\frac{\psi_{1}}{\varepsilon_{1}}\right)\right|, \\
\left|L_{f}^{3} h_{2}(\mathbf{x})\right| \leq k_{2}\left|\tanh \left(\frac{\psi_{2}}{\varepsilon_{2}}\right)\right| \\
\left|L_{f}^{3} h_{3}(\mathbf{x})\right| \leq k_{3}\left|\tanh \left(\frac{\psi_{3}}{\varepsilon_{3}}\right)\right| \\
\left|L_{f}^{2} h_{4}(\mathbf{x})\right| \leq k_{4}\left|\tanh \left(\frac{\psi_{4}}{\varepsilon_{4}}\right)\right|
\end{array}\right.
$$

where $k_{1}, k_{2}, k_{3}, k_{4}, \varepsilon_{1}, \varepsilon_{2}, \varepsilon_{3}$, and $\varepsilon_{4}$ are all positive constants, and the values of constants $\varepsilon_{1}, \varepsilon_{2}, \varepsilon_{3}$, and $\varepsilon_{4}$ are far less than 1 .

Remark 2. The forms of output functions $y_{1}, z_{1}, w_{1}$, and $m_{1}$ can also be set as follows:

$$
\left\{\begin{array}{l}
y_{1}=\delta_{m}, \\
z_{1}=\delta_{L}, \\
w_{1}=I_{\mathrm{dc}}^{2}-1, \\
m_{1}=V_{L}-1 .
\end{array}\right.
$$

That is to say, if we let $L_{1}=1, L_{2}=1, L_{3}=1$, and $L_{4}=1$ in outputs (2), we can get outputs (51). However, from the above input-output feedback linearization derivation process, it can be obtained that $\left|L_{f}^{3} h_{1}(\mathbf{x})\right| \propto L_{1},\left|L_{f}^{3} h_{2}(\mathbf{x})\right| \propto L_{2}$, $\left|L_{f}^{3} h_{3}(\mathbf{x})\right| \propto L_{3}$, and $\left|L_{f}^{2} h_{4}(\mathbf{x})\right| \propto L_{4}$. Considering assumption (50), the reason why the outputs are set to (2) instead of (51) is that the constant parameters $L_{1}, L_{2}, L_{3}$, and $L_{4}$ can be used to limit the amplitude of four system functions $\left|L_{f}^{3} h_{1}(\mathbf{x})\right|,\left|L_{f}^{3} h_{2}(\mathbf{x})\right|,\left|L_{f}^{3} h_{3}(\mathbf{x})\right|$, and $\left|L_{f}^{2} h_{4}(\mathbf{x})\right|$ so that the values of parameters $k_{1}, k_{2}, k_{3}$, and $k_{4}$ are not too large. Since parameters $L_{1}, L_{2}, L_{3}$, and $L_{4}$ play the role of limiting system function amplitudes, the values of these four parameters should be far less than 1 .

Theorem 1. Let the system functions $L_{f}^{3} h_{1}(\mathbf{x}), L_{f}^{3} h_{2}(\mathbf{x})$, $L_{f}^{3} h_{3}(\mathbf{x})$, and $L_{f}^{2} h_{4}(\mathbf{x})$ satisfy assumptions (50). If the control inputs for system (1) are designed as in (52), the four macrovariables $\psi_{1}, \psi_{2}, \psi_{3}$, and $\psi_{4}$ will asymptotically reach the invariant manifolds $\psi_{1}=0, \psi_{2}=0, \psi_{3}=0$, and $\psi_{4}=0$.

$$
\left\{\begin{array}{l}
u_{\mathrm{es} 1}=-\frac{1}{L_{g_{\mathrm{es} 1}} L_{f}^{2} h_{1}(\mathbf{x})}\left[k_{1} \cdot \tanh \left(\frac{\psi_{1}}{\varepsilon_{1}}\right)+\alpha_{2} y_{3}+\alpha_{1} y_{2}+\frac{e^{\left|\psi_{1}\right|} \cdot \psi_{1}}{T_{1}}\right], \\
u_{\mathrm{ST} 1}=-\frac{1}{L_{g_{\mathrm{ST} 1}} L_{f}^{2} h_{2}(\mathbf{x})}\left[k_{2} \cdot \tanh \left(\frac{\psi_{2}}{\varepsilon_{2}}\right)+\beta_{2} z_{3}+\beta_{1} z_{2}+\frac{e^{\left|\psi_{2}\right|} \cdot \psi_{2}}{T_{2}}\right], \\
u_{\mathrm{ST} 2}=-\frac{1}{L_{g_{\mathrm{ST} 2}} L_{f}^{2} h_{3}(\mathbf{x})}\left[k_{3} \cdot \tanh \left(\frac{\psi_{3}}{\varepsilon_{3}}\right)+\gamma_{2} w_{3}+\gamma_{1} w_{2}+\frac{e^{\left|\psi_{3}\right|} \cdot \psi_{3}}{T_{3}}\right], \\
u_{\mathrm{es} 2}=-\frac{1}{L_{g_{\mathrm{es} 2} L_{f} h_{4}(\mathbf{x})}}\left[k_{4} \cdot \tanh \left(\frac{\psi_{4}}{\varepsilon_{4}}\right)+\eta_{1} m_{2}+\frac{e^{\left|\psi_{4}\right|} \cdot \psi_{4}}{T_{4}}\right]
\end{array}\right.
$$

Proof. We first prove that macrovariable $\psi_{1}$ can reach the invariant manifold $\psi_{1}=0$ under the control of $u_{\mathrm{es} 1}$ in (52). Construct a Lyapunov function $V_{1}=\psi_{1}^{2} / 2$, combine (14) and
(46) with the control input $u_{\mathrm{es} 1}$ in (52), and consider assumption (50); the derivative of $V_{1}$ with respect to time can be obtained as follows: 


$$
\begin{aligned}
\dot{V}_{1} & =\psi_{1} \cdot \dot{\psi}_{1}=\psi_{1} \cdot\left[L_{f}^{3} h_{1}(\mathbf{x})+L_{g_{\mathrm{es} 1}} L_{f}^{2} h_{1}(\mathbf{x}) \cdot u_{\mathrm{es} 1}+\alpha_{2} y_{3}+\alpha_{1} y_{2}\right], \\
& =\psi_{1} \cdot L_{f}^{3} h_{1}(\mathbf{x})+\psi_{1} \cdot\left[L_{g_{\mathrm{es} 1}} L_{f}^{2} h_{1}(\mathbf{x}) \cdot u_{\mathrm{es} 1}+\alpha_{2} y_{3}+\alpha_{1} y_{2}\right], \\
& \leq\left|\psi_{1}\right| \cdot k_{1}\left|\tanh \left(\frac{\psi_{1}}{\varepsilon_{1}}\right)\right|+\psi_{1} \cdot\left[L_{g_{\mathrm{es} 1}} L_{f}^{2} h_{1}(\mathbf{x}) \cdot u_{\mathrm{es} 1}+\alpha_{2} y_{3}+\alpha_{1} y_{2}\right], \\
& =\psi_{1} \cdot\left[k_{1} \tanh \left(\frac{\psi_{1}}{\varepsilon_{1}}\right)+L_{g_{\mathrm{es} 1}} L_{f}^{2} h_{1}(\mathbf{x}) \cdot u_{\mathrm{es} 1}+\alpha_{2} y_{3}+\alpha_{1} y_{2}\right], \\
& =\frac{-e^{\left|\psi_{1}\right|} \cdot \psi_{1}^{2}}{T_{1}} \leq 0 .
\end{aligned}
$$

It shows from (53) that macrovariable $\psi_{1}$ reaches the invariant manifold $\psi_{1}=0$ under the control input $u_{\mathrm{es} 1}$ in (52). When the macrovariable reaches the invariant manifold, the first output of system (1) is controlled.

The proof process of macrovariables $\psi_{2}, \psi_{3}$, and $\psi_{4}$ reaching the invariant manifold $\psi_{2}=0, \psi_{3}=0$, and $\psi_{4}=0$ under control inputs $u_{\mathrm{ST} 1}, u_{\mathrm{ST} 2}$, and $u_{\mathrm{es} 2}$ in (52), respectively, is similar to the derivation process in (53), and there is no further proof here. Obviously, compared with inputs in (49), control inputs in (52) do not contain complex system functions $L_{f}^{3} h_{1}(\mathbf{x}), L_{f}^{3} h_{2}(\mathbf{x}), L_{f}^{3} h_{3}(\mathbf{x})$, and $L_{f}^{2} h_{4}(\mathbf{x})$; then, controllers in (52) are more practical.

\section{Simulation Results}

The effectiveness of the proposed control scheme is verified by numerical simulation using MATLAB software. Values of control parameters are selected as follows: $T_{\mathrm{es}}=1, K_{\mathrm{es} 1}=1$, $K_{\mathrm{es} 2}=1, \alpha_{1}=16, \alpha_{2}=8, \beta_{1}=16, \beta_{2}=8, \gamma_{1}=16, \gamma_{2}=8$, $\eta_{1}=5, L_{1}=0.2, L_{2}=0.2, L_{3}=0.2, L_{4}=0.2, \quad k_{1}=500$, $k_{2}=500, k_{3}=500, k_{4}=500, \varepsilon_{1}=0.2, \varepsilon_{2}=0.2, \varepsilon_{3}=0.2$, $\varepsilon_{4}=0.2, T_{1}=0.5, T_{2}=0.5, T_{3}=0.5$, and $T_{4}=0.5$. All values of the aforementioned parameters are in per unit except for angles, which are in degrees. After the four controllers (52) are put into operation, time responses of state variables of the whole controlled power system (1) are given as in Figures 3 and 4 . It can be seen from Figure 3 that chaotic oscillation in the four-dimensional power system is effectively controlled, and it can be seen from Figure 4 that the state variables of the STATCOM device and energy storage device are also restored to the equilibrium state, and then the whole controlled power system can be restored to the stable operation state.

Figure 5 displays the state evolution process of the uncontrolled power system and the controlled power system in the phase plane. As shown in Figure 5, the uncontrolled power system gradually forms strange attractors in the $\delta_{m}-$ $\omega$ phase plane and $\delta_{L}-V_{L}$ phase plane. While the controlled system is different, the state of the whole controlled power system gradually evolves into fixed point $B$ through initial

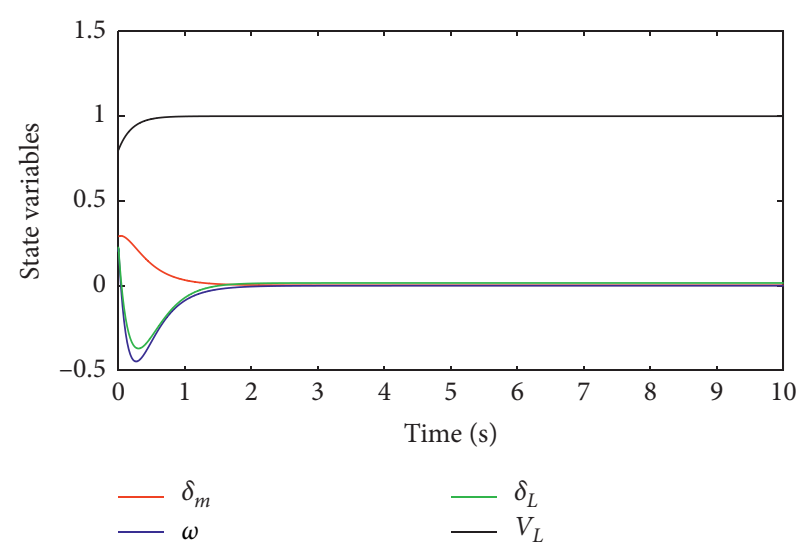

FIgURE 3: Time responses of the four-dimensional power system.
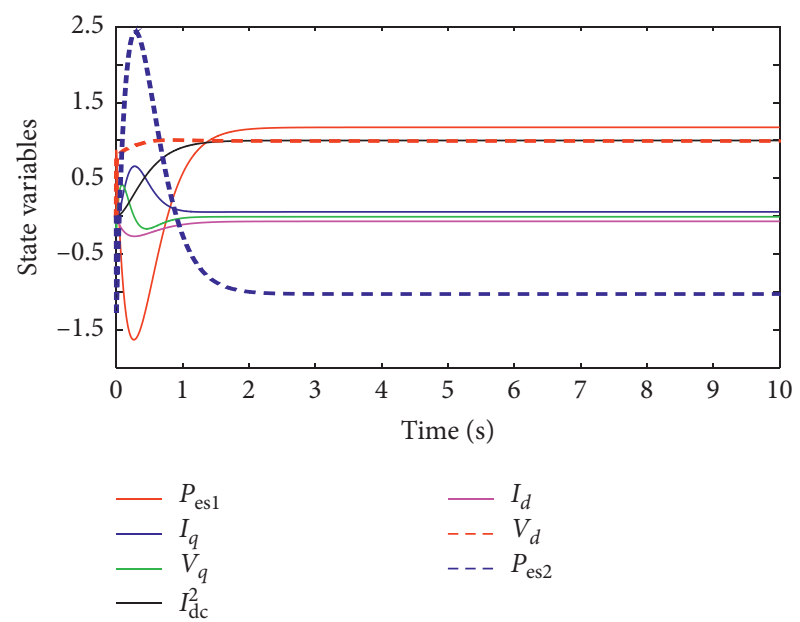

Figure 4: Time responses of the two control devices.

point $A$ in the $\delta_{m}-\omega$ phase plane, and the system state gradually evolves into fixed point $D$ through initial point $C$ in the $\delta_{L}-V_{L}$ phase plane. All the above simulation results verify the effectiveness of the proposed controllers.

In order to highlight the superiority of the proposed fast synergetic control method (47) over the traditional synergetic control method (48), the time responses of macrovariable (46) under the controllers given by the two methods are shown in Figure 6. In Figure 6, $\psi_{1}, \psi_{2}, \psi_{3}$, and $\psi_{4}$, respectively, represent the macrovariables under the fast synergetic controllers (52), while $\psi_{\operatorname{tr} 1}, \psi_{\operatorname{tr} 2}, \psi_{\operatorname{tr} 3}$, and $\psi_{\operatorname{tr} 4}$, respectively, represent the macrovariables under the traditional synergetic controller (the expression of the traditional synergetic controller can be obtained by letting $e^{\left|\psi_{i}\right|}=1(i=$ $1,2,3,4)$ in $(52))$. It can be seen from Figure 6 that the evolution speed of each macrovariable under the proposed fast synergetic controller is obviously faster than that under the traditional synergetic controller. 


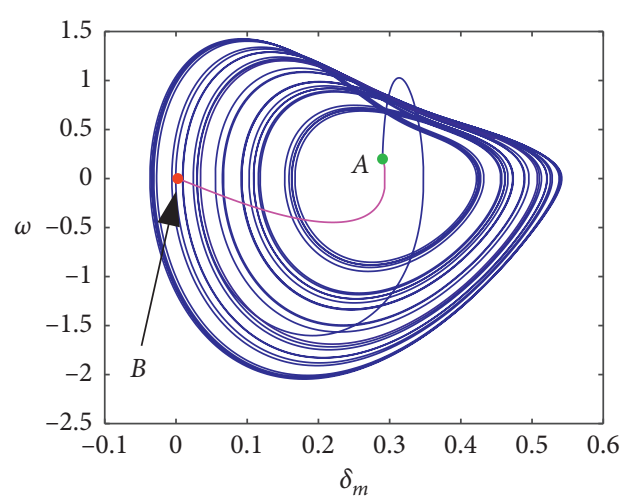

(a)

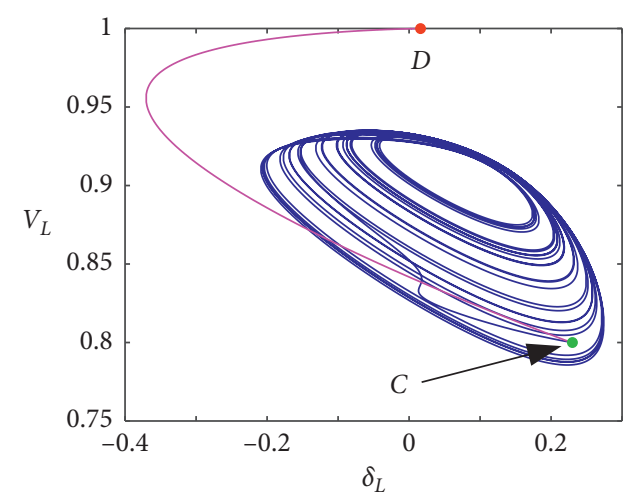

(b)

FIGURE 5: Evolution diagram of the system state in the phase plane.

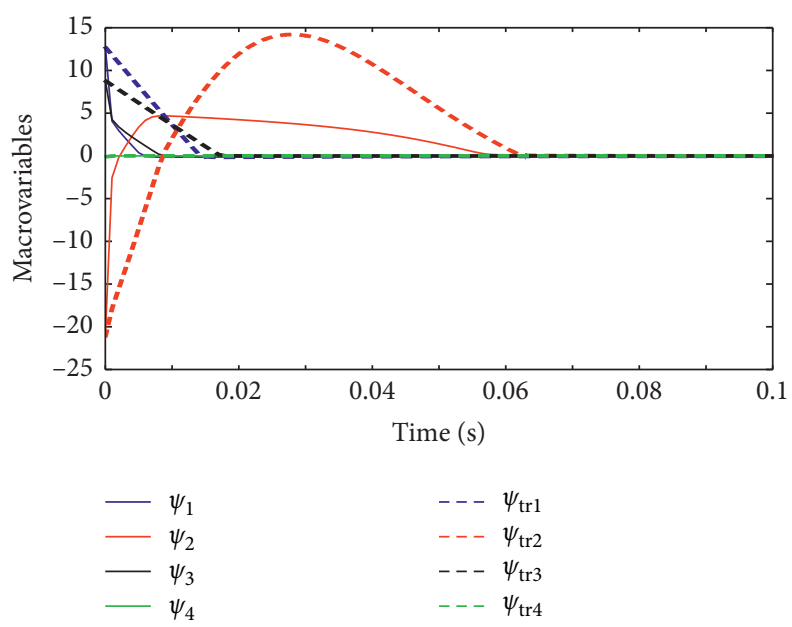

Figure 6: Time responses of macrovariables under the proposed fast synergetic controller and the traditional synergetic controller.

\section{Conclusions}

(1) Aiming at chaos control of a three-bus power system, a nonlinear dynamic system model of the complex controlled power system is constructed. The nonlinear system is linearized step by step, the control problem of the nonlinear power system is transformed into controlling linear systems, and a fast synergetic control scheme is proposed to suppress the chaotic oscillation in the power system.

(2) Although the power system dynamic model with the current source converter-based STATCOM device and energy storage device is only for the four-dimensional power system, the coupling method of the dynamic system can be extended to other three-bus power systems to build other controlled power system dynamic models and put forward similar control methods. Although only the current source converter-based STATCOM device is studied and its chaos control inputs are designed, the voltage source converter-based STATCOM device can also be used to build a similar dynamic model for controlling chaos in the three-bus power system and propose a similar control method.

(3) Different from the widely used input-output feedback linearization-based sliding mode control, the proposed input-output feedback linearizationbased fast synergetic control scheme can provide continuous control laws for the controlled power system, and then the proposed control method has advantages in overcoming the chattering problem. Compared with the traditional synergetic control method, exponential terms are added to the coefficient terms of dynamic equations satisfied by the macrovariables, and the coefficient terms grow exponentially when the system state is far away from the invariant manifolds; then, the proposed fast synergetic control scheme significantly improves the rapidity of traditional synergetic control.

The results of the paper show that chaos control in the power system is ultimately attributed to the control of its active power and reactive power, and the unified power flow 
controller (UPFC) has the ability to regulate active and reactive power at the same time. Therefore, UPFC can be considered to control chaos in the power system in our future work.

\section{Data Availability}

The data used to support the findings of this study are included within this article.

\section{Conflicts of Interest}

The authors declare no conflicts of interest.

\section{Acknowledgments}

This work was supported in part by the National Natural Science Foundation of China (Grant no. 51877162).

\section{References}

[1] B. H. Wang, C. W. Yang, and Q. Zhang, "Summary of bifurcation and chaos research in electric power system," Transactions of China Electrotechnical Society, vol. 20, no. 7, pp. 1-10, 2005, in Chinese.

[2] I. Dobson and H.-D. Chiang, "Towards a theory of voltage collapse in electric power systems," Systems \& Control Letters, vol. 13, no. 3, pp. 253-262, 1989.

[3] H. D. Hsiao-Dong Chiang, C. W. Chih-Wen Liu, P. P. Varaiya, F. F. Wu, and M. G. Lauby, "Chaos in a simple power system," IEEE Transactions on Power Systems, vol. 8, no. 4, pp. 1407-1417, 1993.

[4] Y. Yu, H. Jia, P. Li, and J. Su, "Power system instability and chaos," Electric Power Systems Research, vol. 65, no. 3, pp. 187-195, 2003.

[5] Z. Jing, D. Xu, Y. Chang, and L. Chen, "Bifurcations, chaos, and system collapse in a three node power system," International Journal of Electrical Power \& Energy Systems, vol. 25, no. 6, pp. 443-461, 2003.

[6] J. Ni, C. Liu, K. Liu, and X. Pang, "Variable speed synergetic control for chaotic oscillation in power system," Nonlinear Dynamics, vol. 78, no. 1, pp. 681-690, 2014.

[7] C. Y. Ma, F. X. Wang, Z. J. Li et al., "Adaptive fixed-time fast terminal sliding mode control for chaotic oscillation in power system," Mathematical Problems in Engineering, vol. 2018, Article ID 5819428, 10 pages, 2018.

[8] J. Ni, L. Liu, C. Liu, X. Hu, and S. Li, "Fast fixed-time nonsingular terminal sliding mode control and its application to chaos suppression in power system," IEEE Transactions on Circuits and Systems II: Express Briefs, vol. 64, no. 2, pp. 151-155, 2017.

[9] J. K. Ni, L. Liu, C. X. Liu et al., "Chattering-free time scale separation sliding mode control design with application to power system chaos suppression," Mathematical Problems in Engineering, vol. 2016, Article ID 5943934, 14 pages, 2016.

[10] M. T. Alrifai and M. Zribi, "Sliding mode control of chaos in a single machine connected to an infinite bus power system," Mathematical Problems in Engineering, vol. 2018, Article ID 2703684, 13 pages, 2018.

[11] D. Wei, X. Luo, and Y. Qin, "Controlling bifurcation in power system based on LaSalle invariant principle," Nonlinear Dynamics, vol. 63, no. 3, pp. 323-329, 2011.
[12] Z. Mohamed, M. T. Alrifai, and S. Nejib, "Control of chaos in a single machine infinite bus power system using the discrete sliding mode control technique," Discrete Dynamics in Nature \& Society, vol. 2018, Article ID 5758324, 14 pages, 2018.

[13] H. Zhao, Y. J. Ma, S. J. Liu et al., "Controlling chaos in power system based on finite-time stability theory," Chinese Physics $B$, vol. 20 , no. 12 , pp. 1-8, 2011.

[14] C.-Y. Ma, J.-H. Liu, and C.-L. Wang, "Chaos of a power system model and its control," Journal of Vibration and Control, vol. 18, no. 14, pp. 2176-2185, 2012.

[15] J. Ni, L. Liu, C. Liu, X. Hu, and T. Shen, "Fixed-time dynamic surface high-order sliding mode control for chaotic oscillation in power system," Nonlinear Dynamics, vol. 86, no. 1, pp. 401-420, 2016.

[16] C. A. Canizares, "Power flow and transient stability models of FACTS controllers for voltage and angle stability studies," in Proceedings of the IEEE Power Engineering Society Winter Meeting, pp. 1447-1454, IEEE, January 2000.

[17] N. Mithulananthan, C. A. Canizares, J. Reeve, and G. J. Rogers, "Comparison of PSS, SVC, and STATCOM controllers for damping power system oscillations," IEEE Transactions on Power Systems, vol. 18, no. 2, pp. 786-792, 2003.

[18] H. Chen, Y. Wang, and R. Zhou, "Analysis of voltage stability enhancement via unified power flow controller," in Proceedings of the International Conference on Power System Technology, pp. 403-408, IEEE, December 2000.

[19] J. Guo, M. L. Crow, and J. Sarangapani, "An improved UPFC control for oscillation damping," IEEE Transactions on Power Systems, vol. 24, no. 1, pp. 288-296, 2009.

[20] Q. Jiang, Z. Zou, Z. Wang, and Y. Cao, "Design of UPFC controller in large-scale power systems based on immune genetic algorithm," Transactions of the Institute of Measurement and Control, vol. 28, no. 1, pp. 15-25, 2006.

[21] Y. Wang, H. Chen, and R. Zhou, "A nonlinear controller design for SVC to improve power system voltage stability," International Journal of Electrical Power \& Energy Systems, vol. 22, no. 7, pp. 463-470, 2000.

[22] Y. Wang, Y. L. Tan, and G. Guo, "Robust nonlinear coordinated generator excitation and SVC control for power systems," International Journal of Electrical Power \& Energy Systems, vol. 22, no. 3, pp. 187-195, 2000.

[23] W. Wei Qiao, R. G. Harley, and G. K. Venayagamoorthy, "Coordinated reactive power control of a large wind farm and a STATCOM using heuristic dynamic programming," IEEE Transactions on Energy Conversion, vol. 24, no. 2, pp. 493-503, 2009.

[24] G. Shahgholian and J. Faiz, "Static synchronous compensator for improving performance of power system: a review," International Review of Electrical Engineering, vol. 5, no. 5, pp. 2333-2342, 2010.

[25] A. K. Jain, K. Joshi, A. Behal, and N. Mohan, "Voltage regulation with STATCOMs: modeling, control and results," IEEE Transactions on Power Delivery, vol. 21, no. 2, pp. 726-735, 2006.

[26] J. F. Zhou, Y. Q. Gu, and S. Q. Wei, "Comprehensive comparative analysis of SVC and STATCOM," Electric Power Automation Equipment, vol. 27, no. 12, pp. 61-64, 2007, in Chinese.

[27] Y. L. Tan, "Analysis of line compensation by shunt-connected FACTS controllers: a comparison between SVC and STATCOM," IEEE Power Engineering Review, vol. 19, no. 8, pp. 57-58, 1999.

[28] Y. Ye, M. Kazerani, and V. H. Quintana, "Current-source converter based STATCOM: modeling and control," IEEE 
Transactions on Power Delivery, vol. 20, no. 2, pp. 795-800, 2005.

[29] D. Dong Shen and P. W. Lehn, "Modeling, analysis, and control of a current source inverter-based STATCOM," IEEE Transactions on Power Delivery, vol. 17, no. 1, pp. 248-253, 2002.

[30] J. Fang, W. Yao, Z. Chen, J. Wen, and S. Cheng, "Design of anti-windup compensator for energy storage-based damping controller to enhance power system stability," IEEE Transactions on Power Systems, vol. 29, no. 3, pp. 1175-1185, 2014.

[31] A. F. Payam, M. N. Hashemnia, and J. Fai, "Robust DTC control of doubly-fed induction machines based on inputoutput feedback linearization using recurrent neural networks," Journal of Power Electronics, vol. 11, no. 5, pp. 719-725, 2011.

[32] H. Li, B. L. Zhang, and R. G. Zhou, "The design of excitation controller for generator by means of the method of direct linearization over wide range," Proceedings of the CSEE, vol. 12, no. 2, pp. 35-41, 1992, in Chinese.

[33] A. B. Hmed, T. Bakir, A. Sakly et al., "Input-output feedback linearization control (IOFLC) for muscle force control by functional electrical stimulation," in Proceedings of the 26th Mediterranean Conference on Control and Automation, pp. 619-624, IEEE, June 2018.

[34] C. P. Zhang, F. Lin, W. C. Song et al., "Nonlinear control of induction motors based on direct feedback linearization," Proceedings of the CSEE, vol. 23, no. 2, pp. 99-102, 2003, in Chinese.

[35] P. H. Li, J. Wang, and F. Wu, "Sub-synchronous control interaction mitigation for DFIGs by sliding mode control strategy based on feedback linearization," Transactions on China Electrotechnical Society, vol. 34, no. 17, pp. 3661-3671, 2019, in Chinese.

[36] J. Y. Le, Y. X. Xie, Q. Z. Hong et al., "Sliding mode control of boost converter based on exact feedback linearization," Proceedings of the CSEE, vol. 31, no. 30, pp. 16-23, 2011, in Chinese.

[37] H. Gong, Y. H. Wang, Y. Li et al., "An input-output feedback linearized sliding mode control for D-STATCOM," Automation of Electric Power Systems, vol. 40, no. 5, pp. 102-108, 2016, in Chinese.

[38] E. Santi, A. Monti, D. Li et al., "Synergetic control for power electronics applications: a comparison with the sliding mode approach," Journal of Circuits, Systems, and Computers, vol. 13, no. 4, pp. 737-760, 2004.

[39] E. Akkari, S. Chevallier, and L. Boillereaux, "Global linearizing control of MIMO microwave-assisted thawing," Control Engineering Practice, vol. 17, no. 1, pp. 39-47, 2009.

[40] T. Ademoye and A. Feliachi, "Reinforcement learning tuned decentralized synergetic control of power systems," Electric Power Systems Research, vol. 86, no. 1, pp. 34-40, 2012.

[41] Z. Jiang and R. A. Dougal, "Synergetic control of power converters for pulse current charging of advanced batteries from a fuel cell power source," IEEE Transactions on Power Electronics, vol. 19, no. 4, pp. 1140-1150, 2004.

[42] S. Djennoune and M. Bettayeb, "Optimal synergetic control for fractional-order systems," Automatica, vol. 49, no. 7, pp. 2243-2249, 2013.

[43] E. Santi, A. Monti, D. H. Donghong Li, K. Proddutur, and R. A. Dougal, "Synergetic control for DC-DC boost converter: implementation options," IEEE Transactions on Industry Applications, vol. 39, no. 6, pp. 1803-1813, 2003.

[44] P. Zhao, W. Yao, J. Wen, L. Jiang, S. Wang, and S. Cheng, "Improved synergetic excitation control for transient stability enhancement and voltage regulation of power systems," International Journal of Electrical Power \& Energy Systems, vol. 68, no. 1, pp. 44-51, 2015.

[45] Z. Jiang, "Design of a nonlinear power system stabilizer using synergetic control theory," Electric Power Systems Research, vol. 79, no. 6, pp. 855-862, 2009. 